C-A/AP/\#112

September 2003

Comments on the Operation of the RHIC CNI Polarimeters

\author{
H. Spinka \\ Argonne National Laboratory
}

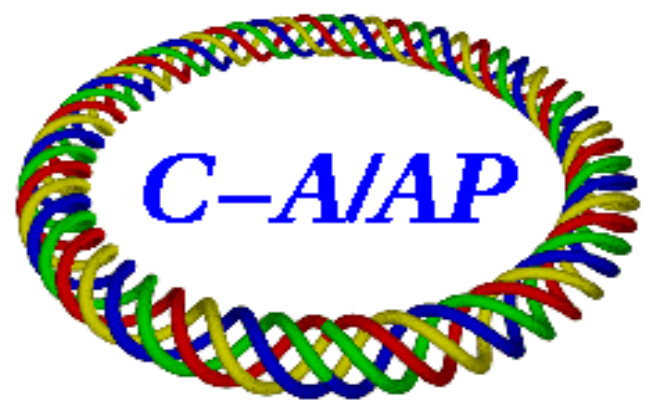

Collider-Accelerator Department Brookhaven National Laboratory

Upton, NY 11973 
$\mathrm{C}-\mathrm{A} / \mathrm{AP} / 112$

September, 2003

\title{
Comments on the Operation of the RHIC CNI Polarimeters
}

\author{
H. Spinka ${ }^{1}$ \\ ${ }^{1}$ High Energy Physics Division \\ Argonne National Laboratory \\ Argonne, Illinois 60439
}

(Dated: September 24, 2003)

\begin{abstract}
The RHIC polarimeter data have exhibited behavior suggesting that systematic effects are present at a level comparable to the statistical uncertainty per run, or $\sim 4 \times 10^{-4}$, in the physics asymmetries and somewhat larger in the luminosity asymmetries. This corresponds to an error in the polarization of approximately $\delta P \sim \pm 0.03$. Two effects are studied that might explain some of the observations, both based on detector rates. The results may explain the systematic effects in both the physics and the luminosity asymmetries. The first effect is caused by the presence of both a background and a good p $+\mathrm{C}$ elastic scattering event in the same passage of a bunch through the polarimeter target. The second effect similarly depends on the presence of multiple $\mathrm{p}+\mathrm{C}$ elastic events from the same bunch. If these rate effects become large enough (or if $\delta P \sim \pm 0.03$ continues and the beam polarization is less than 0.60), they could prevent determination of the beam polarization to $\delta P / P= \pm 5 \%$ unless a correction can be determined to the measured asymmetries.
\end{abstract}




\section{INTRODUCTION}

Possible explanations for some of the problems encountered with the CNI polarimeters in RHIC during the 2000/2001 run period [1,2] are given. These problems include:

- unexpected nonzero up-down and cross ratio physics asymmetries, $\epsilon_{p h y s, Y 45}$ and $\epsilon_{\text {phys }, C R 45}$, respectively.

- fluctuations outside statistics for the least squares fit to the data from the 6 detectors in each RHIC polarimeter.

- differences outside statistical errors of the three luminosity asymmetries: $\epsilon_{\text {lum,1-4 }}$, $\epsilon_{\text {lum }, 2-5}$, and $\epsilon_{\text {lum }, 3-6}$.

- larger deviations from zero than expected from statistical uncertainties when bunch spin directions were artificially reassigned to give zero net polarization to "+" and "_" sets of bunches.

On the basis of these observations, it is thought that the size of systematic errors in physics asymmetries from the CNI polarimeters is comparable to, or somewhat smaller than, the statistical uncertainties for individual runs, or about $0.3-0.4 \times 10^{-3}$. The goal of this note is to discuss mechanisms that may explain some of the problems noted above.

\section{THE RHIC CNI POLARIMETERS}

The RHIC CNI polarimeters consist of six sets of silicon strips in detectors as shown in Fig. 1. Detectors 2 and 5 are in the horizontal plane on opposite sides of the beam, with the strips having a narrow $\theta$ and a wider $\phi$ acceptance. The other four detectors are at $\pm 45^{\circ}$ to the horizontal plane with strips having a narrow $\phi$ and a wider $\theta$ acceptance. All detectors viewed a thin, vertical carbon ribbon target, which intercepted only a small fraction of the RHIC beam. For the 2001/2002 run, 8 strips in each detector were instrumented with waveform digitizers (WFD), but there were few counts in two of these eight strips for detectors 2 and 5 .

The waveform digitizers and associated electronics select events according to the signal amplitude (ADC) and timing (TDC) relative to the mean time of a bunch passing the 


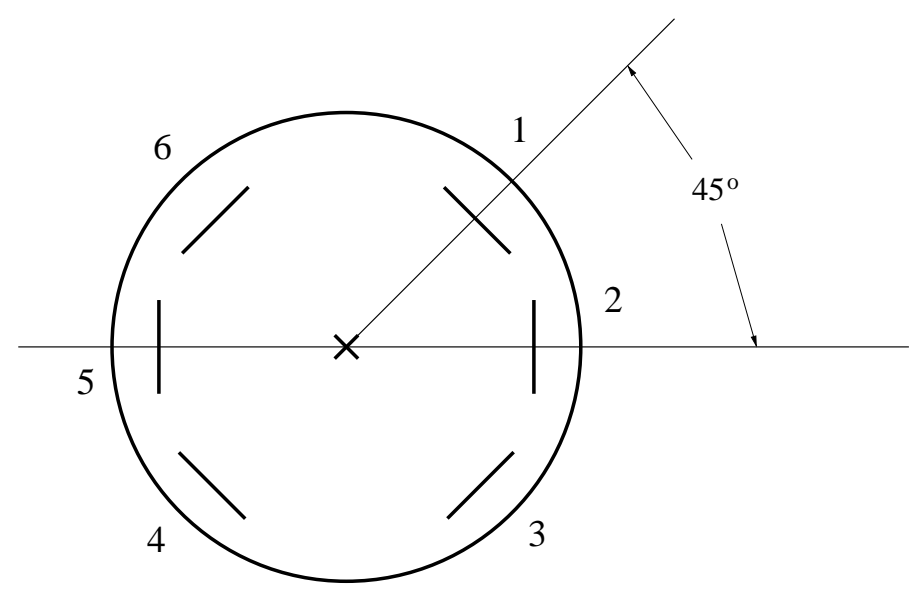

FIG. 1: Layout of the six silicon strip detectors for the RHIC CNI polarimeters, with the beam going into the page. Detectors 1, 2, and 3 are considered "left" and detectors 4, 5, and 6 are "right". This agrees with the standard polarization sign convention that positive analyzing power corresponds to more forward scattered particles to the left than to the right, since the detectors are located to observe the recoil carbons.

polarimeter target. The amplitude must pass a lower threshold cut, and the ADC value is chosen to be the maximum within the WFD time range. The TDC value corresponds to the timing (earlier than the ADC peak) when the amplitude drops below a fixed fraction of the maximum $(\sim 0.25)$. A "banana cut" was applied in the electronics, shown schematically in Fig. 2. The events passing the cuts were recorded separately for each strip and bunch, and for each circuit around RHIC with period $(213 \mathrm{nsec}) \cdot(60$ bunches $) \cong 13 \mu$ sec. Either zero or one event was recorded per passage of each bunch.

Normal data taking runs recorded the number of good events in scalers; backgrounds were not measured. Typical runs collected $20 \mathrm{M}$ events that satisfied the banana cut from the six detectors, and they lasted 30 sec to over 2 min. Other runs recorded the ADC and TDC values for events, and were used to set the banana cut parameters for each strip. 


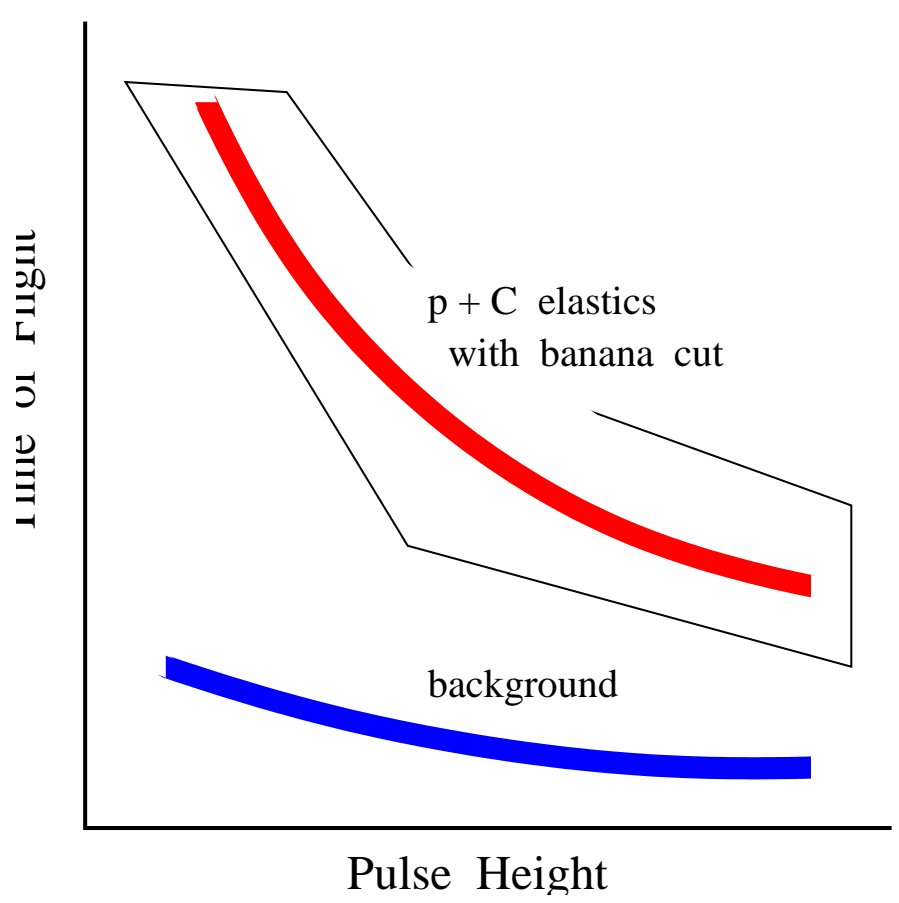

FIG. 2: Schematic showing the flight time and pulse height correlation for events detected in the CNI polarimeters. The band of elastic recoil carbons is shown, together with the "banana cut" applied to the data with the polarimeter electronics. The background extends throughout the plot, including under the $\mathrm{p}+\mathrm{C}$ elastics, but is often concentrated in a band as shown.

\section{BASIC POLARIMETER FORMULAE}

For (approximately) symmetric sets of detectors on opposite sides of the beam ( $\mathrm{L}=$ left, $\mathrm{R}=$ right), the number of counts recorded in some time for idealized detectors will be

$$
\begin{aligned}
& N_{L}^{\uparrow}=N_{0} B^{\uparrow} d \Omega_{L}\left(1+P^{\uparrow} A_{L}\right) \\
& N_{L}^{\downarrow}=N_{0} B^{\downarrow} d \Omega_{L}\left(1-P^{\downarrow} A_{L}\right) \\
& N_{R}^{\uparrow}=N_{0} B^{\uparrow} d \Omega_{R}\left(1-P^{\uparrow} A_{R}\right) \\
& N_{R}^{\downarrow}=N_{0} B^{\downarrow} d \Omega_{R}\left(1+P^{\downarrow} A_{R}\right),
\end{aligned}
$$

where the integrated beam intensities are $B^{\uparrow}$ and $B^{\downarrow}$, and the beam polarizations are $P^{\uparrow}$ and $P^{\downarrow}$ for beam polarization directions up $(\uparrow)$ and down $(\downarrow)$, respectively. The analyzing powers for the $L$ and $R$ detectors are $A_{L}$ and $A_{R}$, and the products of solid angle and efficiency are $d \Omega_{L}$ and $d \Omega_{R}$. A normalization factor, $N_{0}$, is dependent on running time, cross section, target thickness, etc. 
There are three asymmetries that can be determined from square root formulae. The most important is the "physics asymmetry":

$$
\begin{aligned}
\epsilon_{\text {phys }} & =\frac{\sqrt{N_{L}^{\uparrow} N_{R}^{\downarrow}}-\sqrt{N_{L}^{\downarrow} N_{R}^{\uparrow}}}{\sqrt{N_{L}^{\uparrow} N_{R}^{\downarrow}}+\sqrt{N_{L}^{\downarrow} N_{R}^{\uparrow}}} \\
& =\frac{1}{2}\left(P^{\uparrow}+P^{\downarrow}\right) \times \frac{1}{2}\left(A_{L}+A_{R}\right)+\text { h.o.t. } \\
& =P A+\text { h.o.t. }
\end{aligned}
$$

where the higher order terms are fourth order in terms of small quantities such as

$$
\begin{aligned}
\epsilon_{A} & =\frac{A_{L}-A_{R}}{A_{L}+A_{R}} \\
\epsilon_{B} & =\frac{B^{\uparrow}-B^{\downarrow}}{B^{\uparrow}+B^{\downarrow}} \\
\epsilon_{d \Omega} & =\frac{d \Omega_{L}-d \Omega_{R}}{d \Omega_{L}+d \Omega_{R}} \\
\epsilon_{P} & =\frac{P^{\uparrow}-P^{\downarrow}}{P^{\uparrow}+P^{\downarrow}} \\
P A & =\frac{1}{2}\left(P^{\uparrow}+P^{\downarrow}\right) \times \frac{1}{2}\left(A_{L}+A_{R}\right) .
\end{aligned}
$$

For the CNI polarimeters, the term $P A$ is actually second order compared to some of the other four quantities in the list above. The $\epsilon_{B}$ and $\epsilon_{d \Omega}$ terms do not contribute to Eq. (2) when using Eqs. (1). The second type of square root asymmetry is the "luminosity asymmetry", $\epsilon_{l u m}$, which is sensitive to $\epsilon_{B}$ :

$$
\begin{aligned}
\epsilon_{\text {lum }} & =\frac{\sqrt{N_{L}^{\uparrow} N_{R}^{\uparrow}}-\sqrt{N_{L}^{\downarrow} N_{R}^{\downarrow}}}{\sqrt{N_{L}^{\uparrow} N_{R}^{\uparrow}}+\sqrt{N_{L}^{\downarrow} N_{R}^{\downarrow}}} \\
& =\epsilon_{B}+P A \epsilon_{A}+\text { h.o.t., }
\end{aligned}
$$

where the higher order terms are now third order in small quantities, but $\epsilon_{d \Omega}$ is not present when using Eqs. (1). The third type of square root asymmetry is the "geometric asymmetry": 


$$
\begin{aligned}
\epsilon_{\text {geom }} & =\frac{\sqrt{N_{L}^{\uparrow} N_{L}^{\downarrow}}-\sqrt{N_{R}^{\uparrow} N_{R}^{\downarrow}}}{\sqrt{N_{L}^{\uparrow} N_{L}^{\downarrow}}+\sqrt{N_{R}^{\uparrow} N_{R}^{\downarrow}}} \\
& =\epsilon_{d \Omega}+P A \epsilon_{P}+\text { h.o.t. }
\end{aligned}
$$

where the higher order terms are also third order in small quantities, and $\epsilon_{B}$ is absent.

The square root asymmetries were applied to a number of quantities from the CNI polarimeter data. Summing counts from detectors 1 and 3 for "left" and detectors 4 and 6 for "right" and applying Eq. (1) gave the X45 physics asymmetry, $\epsilon_{\text {phys }, X 45}$. This should be equal to $\sin 45^{\circ}$ times the asymmetry from detectors 2 and $5, \epsilon_{p h y s, 2-5}$. In a similar manner, summing detectors 1 and 6 for "up" and 3 and 4 for "down" produced the Y45 asymmetry, $\epsilon_{p h y s, Y 45}$. Since the nominal beam polarization direction was vertical, $\epsilon_{p h y s, Y 45}$ was expected to vanish. Finally, the cross ratio (CR45) asymmetries used detectors 1 and 4 for "left" and 3 and 6 for "right" to produce $\epsilon_{p h y s, C R 45}$. For any spin direction, $\epsilon_{p h y s, C R 45}$ should be zero.

Luminosity asymmetries were computed for each pair of detectors on opposite sides of the beam, 1-4, 2-5, and 3-6. These are denoted $\epsilon_{l u m, 1-4}, \epsilon_{l u m, 2-5}$, and $\epsilon_{l u m, 3-6}$. Since all six detectors viewed the same target at the same time, these three luminosity asymmetries should agree within statistical uncertainties, as indicated in Eq. (4). For the RHIC CNI polarimeters, the correction term $P A \epsilon_{A}$ is expected to be smaller than $\left(\mathrm{PA} \sim 2 \times 10^{-3}\right) \cdot\left(\epsilon_{A} \sim\right.$ $0.05) \sim 10^{-4}$ in magnitude, or a factor 3-4 smaller than statistical errors.

Finally, a least squares fit was made to the data from the six detectors to determine the $\mathrm{X}$ and $\mathrm{Y}$ physics asymmetries. The chi-squared from the fit was evaluated to test for consistency of the data.

\section{RATE EFFECTS IN THE CNI POLARIMETERS}

This section will describe a pair of mechanisms that could produce the observed systematic effects in the polarimeter data. The actual situation is much more complicated than can be accurately modeled in this note. Therefore, simplifying assumptions will be made in order to make estimates of the size of systematic effects in $\epsilon_{p h y s}$ and $\epsilon_{\text {lum }}$. Rough agreement with observations would suggest that further, detailed investigation is warranted. 
Consider two possible sources of rate effects in the RHIC polarimeters. In the first case, $\alpha$ 's or some background process could be present at the "same time" as a true p + C elastic scattering event. That is, they would be recorded in the same WFD readin and could both come from the same bunch interacting in the target. The data indicate such background events usually occur with small TDC values and a range of signal sizes (ADC). The combined event would then have a smaller TDC value than typical $\mathrm{p}+\mathrm{C}$ events, and thus would likely fail the banana cut, resulting in the loss of a good $\mathrm{p}+\mathrm{C}$ event. For this analysis, it will be assumed that a good event is always lost when a background event is also present. A detailed simulation is required to evaluate the true impact of the background.

The fraction of time there would be a background event from a bunch hitting the polarimeter target (or occupancy) will be denoted $f_{B}$. It will be assumed initially that this fraction is not spin dependent, but may differ from detector to detector due to different dead layers in the silicon strips, etc. The fractions for the left and right detectors and up and down beam spins are $f_{B L}^{\uparrow}, f_{B L}^{\downarrow}, f_{B R}^{\uparrow}$, and $f_{B R}^{\downarrow}$. These fractions will reduce the number of good events detected.

The second type of rate effect occurs if two p + C elastic events occur at the "same time". In this case, the combined event would have a large TDC value and a larger ADC value than for a single, typical $\mathrm{p}+\mathrm{C}$ elastic event. It will be assumed that this event is counted by the polarimeter electronics, resulting in the net loss of one $\mathrm{p}+\mathrm{C}$ event. It can be shown that the size of the calculated effect on the asymmetries doubles if no event is counted for such combined events. Again, a detailed simulation is required to accurately estimate this effect.

The fraction of the time there would be a good $\mathrm{p}+\mathrm{C}$ elastic event from a bunch striking the polarimeter target will depend on both the detector and spin direction. These will be denoted $f_{L}^{\uparrow}, f_{L}^{\downarrow}, f_{R}^{\uparrow}$, and $f_{R}^{\downarrow}$, with an average of $f_{T}$.

Including the two rate effects described above into Eqs. (1), then the number of events in the RHIC polarimeters will be:

$$
\begin{aligned}
& N_{L}^{\uparrow}=N_{0} B^{\uparrow} d \Omega_{L}\left(1+P^{\uparrow} A_{L}\right) \cdot\left(1-f_{B L}^{\uparrow}-f_{L}^{\uparrow}\right) \\
& N_{L}^{\downarrow}=N_{0} B^{\downarrow} d \Omega_{L}\left(1-P^{\downarrow} A_{L}\right) \cdot\left(1-f_{B L}^{\downarrow}-f_{L}^{\downarrow}\right) \\
& N_{R}^{\uparrow}=N_{0} B^{\uparrow} d \Omega_{R}\left(1-P^{\uparrow} A_{R}\right) \cdot\left(1-f_{B R}^{\uparrow}-f_{R}^{\uparrow}\right) \\
& N_{R}^{\downarrow}=N_{0} B^{\downarrow} d \Omega_{R}\left(1+P^{\downarrow} A_{R}\right) \cdot\left(1-f_{B R}^{\downarrow}-f_{R}^{\downarrow}\right) .
\end{aligned}
$$


It is assumed that the effects of bunches with two good $\mathrm{p}+\mathrm{C}$ elastic scatters and a background event are negligible in Eqs. (6). Inserting these revised expressions into Eqs. (2) and (4) gives to second order in $f_{i}$ :

$$
\begin{aligned}
\epsilon_{\text {phys }} \cong & P A+\left(f_{L}^{\downarrow}+f_{R}^{\uparrow}-f_{L}^{\uparrow}-f_{R}^{\downarrow}\right)\left(1+2 f_{T}+2 f_{B}\right) / 4+ \\
& \left(f_{B L}^{\downarrow}+f_{B R}^{\uparrow}-f_{B L}^{\uparrow}-f_{B R}^{\downarrow}\right)\left(1+2 f_{T}+2 f_{B}\right) / 4+ \\
& {\left[\left(f_{L}^{\uparrow}+f_{B L}^{\uparrow}\right)\left(f_{R}^{\downarrow}+f_{B R}^{\downarrow}\right)-\left(f_{L}^{\downarrow}+f_{B L}^{\downarrow}\right)\left(f_{R}^{\uparrow}+f_{B R}^{\uparrow}\right)\right] / 4+\text { h.o.t. } } \\
\epsilon_{\text {lum }} \cong & \epsilon_{B}+P A \epsilon_{A}+\left(f_{L}^{\downarrow}+f_{R}^{\downarrow}-f_{L}^{\uparrow}-f_{R}^{\uparrow}\right)\left(1+2 f_{T}+2 f_{B}\right) / 4+ \\
& \left(f_{B L}^{\downarrow}+f_{B R}^{\downarrow}-f_{B L}^{\uparrow}-f_{B R}^{\uparrow}\right)\left(1+2 f_{T}+2 f_{B}\right) / 4+ \\
& {\left[\left(f_{L}^{\uparrow}+f_{B L}^{\uparrow}\right)\left(f_{R}^{\uparrow}+f_{B R}^{\uparrow}\right)-\left(f_{L}^{\downarrow}+f_{B L}^{\downarrow}\right)\left(f_{R}^{\downarrow}+f_{B R}^{\downarrow}\right)\right] / 4+\text { h.o.t. } }
\end{aligned}
$$

It is anticipated that the magnitude of the corrections will generally be smaller for the physics than the luminosity asymmetry, because of the expected partial cancellation of $f_{L}^{\uparrow}$ and $f_{R}^{\uparrow}$ and of $f_{L}^{\downarrow}$ and $f_{R}^{\downarrow}$ in $\epsilon_{\text {phys }}$, etc. Equations (7) and (8) are the important results from this note, and should be used to evaluate whether such rate effects are actually affecting the asymmetries from the polarimeter data at a significant level. They assume the occupancies are small, comparable to $\epsilon_{A}, \epsilon_{B}, \epsilon_{d \Omega}$, and $\epsilon_{P}$, but perhaps significantly larger in magnitude than $P A$. Also, whereas $\epsilon_{p h y s}$ was independent of $\epsilon_{B}$ and $\epsilon_{d \Omega}$ using Eqs. (1), this will generally no longer be true using Eqs. (6).

\section{ESTIMATES OF THE SIZE OF RATE EFFECTS}

In order to make numerical estimates, an additional assumption is required. It will be assumed that the fractions $f_{j}$ are proportional to the observed counts $N_{j}$; this fails if not all silicon strips are working or recorded in all detectors. Then

$$
\begin{aligned}
f_{L}^{\uparrow} \cong f_{T} N_{0} B^{\uparrow} d \Omega_{L}\left(1+P^{\uparrow} A_{L}\right) / N_{0} B d \Omega \\
f_{L}^{\downarrow} \cong f_{T} N_{0} B^{\downarrow} d \Omega_{L}\left(1-P^{\downarrow} A_{L}\right) / N_{0} B d \Omega \\
f_{R}^{\uparrow} \cong f_{T} N_{0} B^{\uparrow} d \Omega_{R}\left(1-P^{\uparrow} A_{R}\right) / N_{0} B d \Omega \\
f_{R}^{\downarrow} \cong f_{T} N_{0} B^{\downarrow} d \Omega_{R}\left(1+P^{\downarrow} A_{R}\right) / N_{0} B d \Omega,
\end{aligned}
$$

where $B=\left(B^{\uparrow}+B^{\downarrow}\right) / 2$ and $d \Omega=\left(d \Omega_{L}+d \Omega_{R}\right) / 2$ are averages. It can be verified that the average of the four quantities in Eq. (9) is indeed $f_{T}$. The background fractions will 
initially be assumed to depend only on the beam intensity and solid angle times efficiency, that is to be spin independent:

$$
\begin{aligned}
f_{B L}^{\uparrow} \cong f_{B} N_{0} B^{\uparrow} d \Omega_{L} / N_{0} B d \Omega \\
f_{B L}^{\downarrow} \cong f_{B} N_{0} B^{\downarrow} d \Omega_{L} / N_{0} B d \Omega \\
f_{B R}^{\uparrow} \cong f_{B} N_{0} B^{\uparrow} d \Omega_{R} / N_{0} B d \Omega \\
f_{B R}^{\downarrow} \cong f_{B} N_{0} B^{\downarrow} d \Omega_{R} / N_{0} B d \Omega
\end{aligned}
$$

where $f_{B}$ is the average of these four quantities. Substituting these into Eqs. (7) and (8) gives:

$$
\begin{gathered}
\epsilon_{\text {phys }} \cong P A\left(1-f_{T}\right)-\left(f_{T}+f_{B}\right) \epsilon_{B} \epsilon_{d \Omega}+\text { h.o.t. } \\
\epsilon_{\text {lum }} \cong \epsilon_{B}\left[1-\left(f_{T}+f_{B}\right)\right]+P A \epsilon_{A}+\text { h.o.t. }
\end{gathered}
$$

It is assumed that $P A$ is second order compared to $\epsilon_{B}, \epsilon_{d \Omega}$, and the occupancies in the equations above. The higher order terms are fourth order in small quantities $\left(\epsilon_{B}, \epsilon_{d \Omega}, f\right)$ in Eq. (11) and third order in Eq. (12). The factors multiplying $P A$ and $\epsilon_{B}$ in Eqs. (11) and (12) are dilutions of the asymmetries due to the greater loss of events for the detector and spin state with the higher rates. This dilution persists even when the detectors have equal solid angles and efficiencies, or $\epsilon_{d \Omega}=0$. On the other hand, the $\left(f_{T}+f_{B}\right) \epsilon_{B} \epsilon_{d \Omega}$ term is a correction specifically for different occupancies when the beam intensities differ and the $d \Omega$ factors are not equal. It does $\underline{\text { NOT }}$ require spin dependence to the occupancy.

Note, the correction $-f_{T} P A$ comes specifically from such spin dependence in the $f_{i}$. If the background likewise has a spin dependence, with left and right analyzing powers $A_{B L}$ and $A_{B R}$, there is no change to Eq. (12) to third order, but Eq. (11) becomes:

$$
\epsilon_{\text {phys }} \cong P A\left(1-f_{T}\right)-\left(f_{T}+f_{B}\right) \epsilon_{B} \epsilon_{d \Omega}-f_{B} P A_{B}+\text { h.o.t. }
$$

where $A_{B}=\left(A_{B L}+A_{B R}\right) / 2$ is the analyzing power of the background reaction(s).

The typical physics asymmetry for the $2001 / 2002$ run was $\epsilon_{\text {phys }} \cong P A \cong 2 \times 10^{-3}$, and luminosity asymmetries were often as large in magnitude as 0.04 , so $\left|\epsilon_{B}\right| \sim 0.04$ will be assumed. (Note, nonzero $\epsilon_{B}$ can result from a different number of $\uparrow$ and $\downarrow$ bunches even if the instantaneous intensities per bunch are all equal. However, for the effects described 
in this note, instantaneous rates are really the important quantities.) Similarly, $\left|\epsilon_{\text {geom }}\right|$ was frequently 0.1 , although this may have been inaccurate if one or more strips were not working in a detector.

Finally, the average fractions $f_{T}$ and $f_{B}$ must be estimated. Typical runs collected $20 \times 10^{6}$ events that passed banana cuts in 6 strips in 6 detectors. Runs were often as short as 30 sec. Of the 60 bunch positions in each ring, only 55 contained beam when taking into account the abort gap, and only 52 had nonzero polarizations. The bunch spacing was 213 nsec, and some strips had twice the count rate of the average [3]. Then

$$
\begin{aligned}
f_{T} & \cong\left(\frac{20 \times 10^{6} \text { events }}{6 \times 6 \text { strips }}\right) \cdot\left(\frac{213 \times 10^{-9} \mathrm{sec} / \text { bunch }}{30 \mathrm{sec} \text { run }}\right) \cdot\left(\frac{60 \text { bunches total }}{52 \text { useful bunches }}\right) \cdot(2 \times \text { av.rate }) \\
& \cong 9.1 \times 10^{-3} .
\end{aligned}
$$

The background occupancy, $f_{B}$, can only be roughly estimated. A portion of the background is apparent in event data taken to adjust the banana cut, shown schematically in Fig. 2. The fraction of events inside the cut varies with time, operating conditions, and strip; the average value over all strips is $\sim 75 \%$ during runs in April 2003. Thus this contribution will be taken to be $\approx 0.3 f_{T}$, part of which is thought to be $\alpha$ 's.

Another source of background has been termed "prompt" events, believed to be higher momentum charged particles, such as $\pi$ 's and K's. These particles could give large pulses in the strips. They are expected to arrive earlier than the elastically scattered carbon recoil particles, but the tail of these signals could still fall within the WFD time range. These tails could produce relatively large $\mathrm{ADC}$ values, but $\mathrm{TDC} \sim 0$. The algorithm to assign the time for the event would use $\mathrm{TDC}=0$ for any carbon recoil detected with pulse height less than about four times the $\mathrm{ADC}$ value from the prompt event at the time of the $\mathrm{p}+\mathrm{C}$ elastic event. Events with $\mathrm{TDC}=0$ are rejected in the polarimeter logic, and the number of them is not presently recorded. It is estimated [3] that they correspond to between 1 and 10 times the number of events passing the banana cut, so the occupancy will be assumed to be $\approx 3 f_{T}$. This gives a total background occupancy of $f_{B} \approx 3.3 f_{T}=0.03$.

With these estimates, the second order correction to $\epsilon_{\text {lum }}$ is

$$
\delta \epsilon_{\text {lum }} \cong\left(f_{T}+f_{B}\right) \epsilon_{B} \sim 1.6 \times 10^{-3}
$$

which is larger than the statistical uncertainty on $\epsilon_{\text {lum }}$. Hence this rate effect may explain 
the observed differences among $\epsilon_{l u m, 1-4}, \epsilon_{l u m, 2-5}$, and $\epsilon_{l u m, 3-6}$. Similarly, the corrections to the physics asymmetry are

$$
\delta \epsilon_{p h y s} \cong\left(f_{T}+f_{B}\right) \epsilon_{B} \epsilon_{d \Omega} \sim 0.16 \times 10^{-3}
$$

and

$$
\delta \epsilon_{\text {phys }}^{\prime} \cong P A f_{T} \sim 0.02 \times 10^{-3}
$$

The former is similar to the associated statistical uncertainties, but the latter is quite small. A third correction, $\delta \epsilon_{\text {phys }}^{\prime \prime} \cong P A_{B} f_{B}$, cannot be estimated since the analyzing power $\left(A_{B}\right)$ is unknown.

\section{DISCUSSION}

Sizable rate problems may arise for the RHIC CNI polarimeters because: 1) the true physics asymmetries are small, 2) the fraction of bunches passing through the target and producing either a good or a background event above threshold in a strip is appreciable, 3) multiple events per strip and bunch passage are not counted, and 4) details of the background event characteristics and the WFD algorithm preferentially reject good events coming "at the same time." Thus, in some sense the polarimeters are not "deadtimeless".

The estimates made in the previous section may not be very accurate because not all silicon strips contributed to the observed counts $N_{i}$ for all runs. Thus, $f_{T}, f_{B}$, and $\epsilon_{d \Omega}$ could have been over- or underestimated. Furthermore, the strips are approximately parallel to the beam for the $\pm 45^{\circ}$ detectors, but are perpendicular for the detectors in the horizontal plane. This could have concentrated the rates in fewer strips in detectors 2 and 5 , enhancing the rate problems.

It is recommended to:

- Estimate the occupancies, $f_{k}$ and $f_{B n}$, for each strip directly from the data. The impact on the physics and luminosity asymmetries should be evaluated using Eqs. (7) and (8). Especially important is the contribution from the prompt events.

- Test for spin dependence of background processes, since a substantial asymmetry could lead to systematic error in the physics asymmetry; see Eq. (13). 
- Evaluate the magnitude of $\epsilon_{A}$ due to differences in silicon dead layer thicknesses and banana cuts. Assuming $\epsilon_{A}$ is less than 0.1 in magnitude, this would confirm that the term $P A \epsilon_{A}$ in Eq. (4) does not affect $\epsilon_{l u m}$ significantly.

- Perform simulations to determine the fraction of events with two or more $\mathrm{p}+\mathrm{C}$ elastic events at the same time that fail the banana cut, as this will increase the impact of these events on the physics and luminosity asymmetries. Similarly, evaluate the number of good events lost when a background and a $\mathrm{p}+\mathrm{C}$ elastic event occur at the same time.

If one or more of the strips had combined occupancy of significantly greater than $1 \%$ $\left(f_{T}+f_{B}>0.01\right)$, an effort should be made to correct the data for future running. It is presently unclear how accurately this correction could be determined, but an increase in beam intensity or decrease in bunch transverse dimensions would increase $f_{T}$ and $f_{B}$. Recall that when $f_{T}=0.05$, the systematic error would be $\delta P / P= \pm 0.05$ without correction (see Eq. (11)), or the goal for accuracy of the RHIC polarimeters. Furthermore, the highest rates are likely to occur for the recoil carbons detected with the lowest 4-momentum transfer squared, -t, where the analyzing power is largest. Thus the effect on the derived beam polarization may be considerably larger than that expected from averages over all strips.

Finally, this sort of rate problem could conceivably cause systematic errors in other polarimeters or detectors. For example, it has been stated that the new AGS CNI polarimeter could achieve a polarization uncertainty of $\delta P= \pm 0.05$ in a single AGS fill with six bunches at the injection energy for RHIC. Assuming similar hardware and analyzing power ( $A \sim 0.013$ ) as for the RHIC CNI polarimeters, then the number of events required would be

$$
N \sim \frac{1}{(0.05 \cdot 0.013)^{2}} \sim 2.4 \times 10^{6} .
$$

Dividing this by $2^{*} 6$ strips for a left and right pair of detectors would give roughly $2 \times 10^{5}$ events per strip. For a 1 sec AGS flattop to perform the measurements and a $3 \mu$ sec time to travel around the AGS, then the average occupancy would be

$$
f_{T} \cong\left(\frac{2 \times 10^{5} / \mathrm{sec}}{\text { strip }}\right) \cdot\left(\frac{3 \times 10^{-6} \mathrm{sec}}{6 \text { bunches }}\right) \cong 0.1
$$

or a $10 \%$ relative systematic error unless corrected; see Eq. (11). The effect may be even larger if some strips have substantially greater rates than average, as occurs in the RHIC 
polarimeters. In addition, the term $-\left(f_{T}+f_{B}\right) \epsilon_{B} \epsilon_{d \Omega}$ could become quite sizable, also leading to a systematic error.

\section{ACKNOWLEDGEMENTS}

The assistance of the AGS and RHIC staff and physicists from many institutions working with the various polarimeters is gratefully acknowledged. This research was supported in part by the U.S. Deaprtment of Energy, Division of High Energy and Nuclear Physics, Contract No. W-31-109-ENG-38.

[1] O. Jinnouchi et al., AIP Conference Proceedings 675, 817 (2003); O. Jinnouchi in "Proceedings of the RIKEN BNL Research Center Workshop", BNL reports BNL-71117-2003 (2003), p 57, 141, 311, and BNL-71118-2003 (2003), p93, and BNL-71300-2003 (2003), p49.

[2] A. Bravar et al., "Analysis of Polarimeter Data for the 2001-2002 RHIC Run", BNL Collider Accelerator Department note C-A/AP/108 (2003).

[3] O. Jinnouchi, private communication. 